\title{
Strategies of Instantaneous Compensation for Three-Phase Four-Wire Circuits
}

\author{
Juan Carlos Montaño Asquerino, Senior Member, IEEE, and Patricio Salmerón Revuelta
}

\begin{abstract}
When the voltage source is not balanced for three-phase four-wire circuits, instantaneous compensation for the instantaneous reactive power does not eliminate the neutral current on the source side. In fact, when the zero-phase voltage of the source exists, none of the present compensation strategies can guarantee the instantaneous elimination of the neutral current in three-phase four-wire systems. Two approaches are distinguished in this paper for instantaneous compensation. The first eliminates the instantaneous reactive current, thus neutral current can still flow. The second eliminates the instantaneous pseudo-reactive current, so that the neutral current component is compensated. In the latter case, a new control strategy is designed to avoid instantaneous power flowing through the compensator. It provides flexibility in compensating for the neutral current in a three-phase four-wire system including the zero-sequence voltage. Finally, simulated and experimental results are obtained to confirm the theoretical properties and show the compensator performance.
\end{abstract}

Index Terms-Active filters, current control, instantaneous active and reactive current, power system control, reactive power control, three-phase systems.

\section{INTRODUCTION}

$\mathbf{F}$ OR three-phase three-wire systems (see Fig. 1), Akagi and coauthors introduced the so-called " $p-q$ theory" [1]. They analyzed the source voltage and current based on the instantaneous value concept. The value of the instantaneous reactive (or imaginary) current is determined at any single time and, hence, the corresponding value of the compensation current. This enables instantaneous compensation (i.e., instantaneous reactive power compensation using switching devices), which theoretically does not requires any energy storage components. Many instantaneous power theories emerged thereby [2]-[5], and a space vector of the instantaneous reactive power was defined as the vector product of the voltage space vector and the current space vector [5], [6]. This power definition is valid for threephase three-wire systems and for three-phase four-wire systems. However, for three-phase four-wire systems, maximum reactive current compensation cannot guarantee elimination of the neutral current. Compensation for the instantaneous reactive power would enable drawing zero-sequence current from the utility if

Manuscript received March 19, 2001. This work was supported by the "Comision Interministerial de Ciencia y Tecnología (CICYT)" of the Spanish Minister of Education and Culture as part of a project covering aspects of Power Quality measurements and instantaneous compensation of nonlinear loads.

J. C. Montaño Asquerino is with IRNAS (CSIC), Sevilla, Spain (e-mail: montano@irnase.csic.es).

P. Salmerón Revuelta is with the Department of Electrical Engineering, University of Huelva, Spain (e-mail: patricio.salmeron@dfaie.uhu.es).

Digital Object Identifier 10.1109/TPWRD.2002.803841

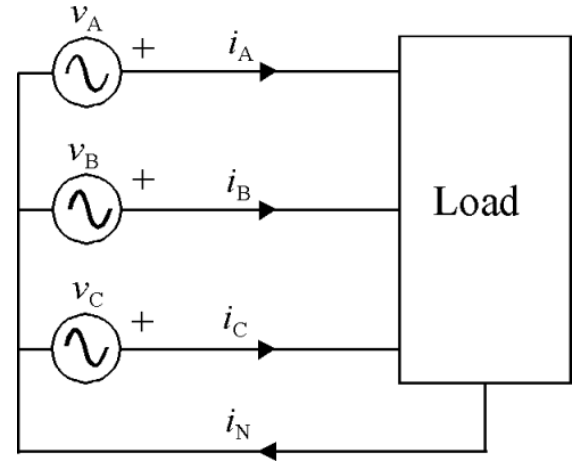

Fig. 1. Three-phase four-wire system configuration.

zero-sequence voltage exists. The authors reviewed the instantaneous currents that are defined according to the instantaneous value concept and indicated the control strategies for reaching instantaneous and full compensation [7], [8]. Compensation for an instantaneous pseudo-reactive power was obtained with the flexibility in compensating for the zero-sequence current- the zero-sequence voltage existing or not. Unfortunately, the instantaneous pseudo-reactive power does not have maximum instantaneous norm.

The introduction of mapping matrices [9] and a holistic approach [10] expanded the $p-q$ theory to three-phase four-wire systems. It provides compensation without energy-storage elements of the zero-sequence current even though the zero-sequence voltage is present in the supply. Although the zero-sequence current is eliminated, the current root-mean-square (RMS) value is not minimal, because instantaneous reactive powers remain uncompensated.

This paper compares the reactive power defined according to the well-established instantaneous power concepts [1]-[6] with the instantaneous pseudo-reactive power defined by the authors [7], [8]. Definitions [1]-[6] lead to the instantaneous compensation of the maximum reactive power/current, including a part (undetermined) of the zero-sequence power. Definitions [7], [8] lead to the instantaneous compensation of the instantaneous pseudo-reactive power, including the total zero-sequence power in all situations.

\section{Instantaneous Active AND Reactive CURRENT VECTORS}

Line currents supplied by three-phase generators (ac source) can be described in vector form according to several established 


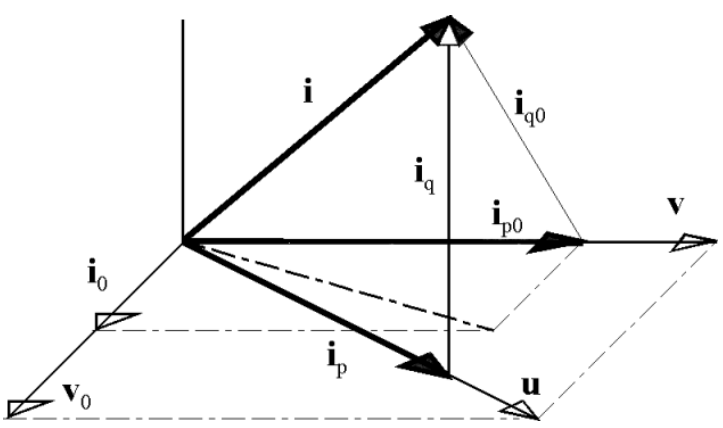

Fig. 2. Instantaneous vectors on $x y z$ coordinates.

theories [3]-[9]. The voltage vector, $\mathbf{u}$, contains as elements instantaneous phase voltages, ${ }^{1}$ and the current vector, $\mathbf{i}$, contains the instantaneous line currents,

$$
\mathbf{u}=\left[\begin{array}{lll}
v_{A} & v_{B} & v_{C}
\end{array}\right]^{T}, \quad \mathbf{i}=\left[\begin{array}{lll}
i_{A} & i_{B} & i_{C}
\end{array}\right]^{T} .
$$

\section{A. First Approach}

According to [3]-[6], the instantaneous active current, $\mathbf{i}_{p}$, is defined as the vector component obtained by projecting the current vector $\mathbf{i}$ on the voltage vector $\mathbf{u}$, as shown in Fig. 2:

$$
\mathbf{i}_{p}=\frac{\mathbf{u}^{T} \cdot \mathbf{i}}{\mathbf{u}^{T} \cdot \mathbf{u}} \mathbf{u}=\frac{p_{u}}{u^{2}} \mathbf{u}
$$

where "." denotes the dot product or scalar product of vectors, and

$$
\begin{aligned}
& p_{u}=\mathbf{u}^{T} \cdot \mathbf{i}=v_{A} i_{A}+v_{B} i_{B}+v_{C} i_{C} \\
& u^{2}=\mathbf{u}^{T} \cdot \mathbf{u}=v_{A} v_{A}+v_{B} v_{B}+v_{C} v_{C} .
\end{aligned}
$$

$p_{u}$ is the instantaneous active power of the three-phase circuit and $u$ the instantaneous magnitude or norm (squared) of the three-phase voltage.

In this case, the instantaneous reactive current vector is the complement [6]:

$$
\mathbf{i}_{q}=\mathbf{i}-\mathbf{i}_{p}
$$

1) Properties: The analysis defined before has the following properties [5], [6].

Property 1: A three-phase current vector $\mathbf{i}$ is always equal to the sum of $\mathbf{i}_{p}$ and $\mathbf{i}_{q}: \mathbf{i} \equiv \mathbf{i}_{p}+\mathbf{i}_{q}$.

Property 2: $\mathbf{i}_{q}$ is orthogonal to $\mathbf{u}$, and $\mathbf{i}_{p}$ is parallel to $\mathbf{u}$ (i.e., $\mathbf{u} \cdot \mathbf{i}_{q} \equiv 0$ and $\left.\mathbf{u} \times \mathbf{i}_{p} \equiv 0\right)$.

Property 3: If $\mathbf{i}_{q}=0$, then the norm $i=\sqrt{ }\left(i_{A}^{2}+i_{B}^{2}+i_{C}^{2}\right)$ becomes minimal for transmitting the same instantaneous active power.

From the definitions and properties listed before, we can reach the following conclusions:

a) The current vector $\mathbf{i}_{p}$ is indispensable for the instantaneous active power $\left(p_{u}\right)$ transmission, whereas $\mathbf{i}_{q}$ does not contribute to it because $p_{u}=\mathbf{u} \cdot \mathbf{i}=\mathbf{u} \cdot \mathbf{i}_{p}$ and $\mathbf{u} \cdot \mathbf{i}_{q} \equiv 0$. Or current

${ }^{1}$ For simplicity, $f$ will be used instead of $f(t)$ for denoting instantaneous quantities.

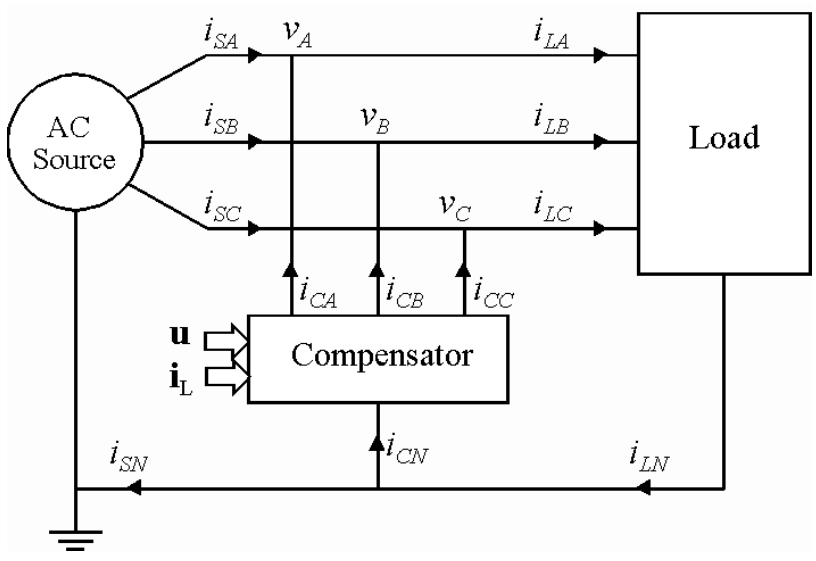

Fig. 3. System configuration of reactive current compensator.

vector $\mathbf{i}_{p}$ transfers to the load instantaneous power $p_{u}$ at voltage $\mathbf{u}$ have the minimum norm (or magnitude) [2].

b) Using active filters without energy storage as compensators, the instantaneous reactive current can be cancelled and the instantaneous active power cannot be changed. The minimum line losses are obtained, ensuring the same instantaneous power consumption of the actual load.

2) Control Strategy: Fig. 3 shows the system configuration of reactive current compensation. The control circuit of the compensator, not shown, senses the current components of the load current vector $\mathbf{i}_{L}=\left[\begin{array}{lll}i_{L A} & i_{L B} & i_{L C}\end{array}\right]^{T}$ for instantaneous compensation. Then the active filter, in parallel with the load, generates the compensation current

$$
\mathbf{i}_{C}=\mathbf{i}_{q}
$$

and the supply current vector verifies

$$
\mathbf{i}_{S}=\mathbf{i}_{L}-\mathbf{i}_{C}=\mathbf{i}_{p}=\frac{p_{u}}{u^{2}} \mathbf{u}
$$

Therefore, as expected, the instantaneous active power generated by the compensator is zero; that is,

$$
p_{C}=\mathbf{u}^{T} \cdot \mathbf{i}_{C}=\mathbf{u}^{T} \cdot \mathbf{i}_{q}=0 .
$$

Under imbalanced supply-voltage conditions, the instantaneous voltage vector, $\mathbf{u}$, contains a zero-sequence voltage component. So (7) clearly shows that the supply current after compensation contains a zero-sequence current component. For three-phase four-wire systems, it implies a neutral current that can still flow into the source side, producing power losses.

\section{B. Second Approach}

The authors developed a general theory of instantaneous power which permits compensation of the zero-sequence current and the reactive current vectors [7], [8], having defined the voltage vectors

$$
\begin{aligned}
\mathbf{v} & =\mathbf{u}-\mathbf{v}_{o} \\
v^{2} & =\mathbf{v}^{T} \cdot \mathbf{v} \\
v_{o}^{2} & =\mathbf{v}_{o}^{T} \cdot \mathbf{v}_{o},
\end{aligned}
$$


shown in Fig. 2, and instantaneous powers

$$
\begin{aligned}
p & =\mathbf{v}^{T} \cdot \mathbf{i} \\
p_{o} & =\mathbf{v}_{o}^{T} \cdot \mathbf{i}_{o}
\end{aligned}
$$

where the zero-sequence voltage

$$
\mathbf{v}_{o}=\frac{v_{o}}{\sqrt{3}}\left[\begin{array}{l}
1 \\
1 \\
1
\end{array}\right], \quad v_{o}=\frac{v_{A}+v_{B}+v_{C}}{\sqrt{3}}
$$

and

$$
p+p_{o}=p_{u}
$$

Analogous relationships are valid for the zero-sequence current.

According to [7], [8], as shown in Fig. 2, the instantaneous pseudoactive current is defined as a current vector

$$
\mathbf{i}_{p 0}=\frac{p_{u}}{v^{2}} \mathbf{v},
$$

which is free of the zero-sequence voltage $\left(\mathbf{u}-\mathbf{v}_{o}\right)$. In this case, the pseudoreactive current vector is the complement

$$
\mathbf{i}_{q 0}=\mathbf{i}-\mathbf{i}_{p 0} .
$$

1) Properties: The analysis defined before has the following properties

Property 1: A three-phase current vector $\mathbf{i}$ is always equal to the sum of $\mathbf{i}_{p 0}$ and $\mathbf{i}_{q 0}: \mathbf{i} \equiv \mathbf{i}_{p 0}+\mathbf{i}_{q 0}$.

Property 2: $\mathbf{v} \times \mathbf{i}_{p 0} \equiv 0$ and

$$
\begin{aligned}
\mathbf{u}^{T} \cdot \mathbf{i}_{q 0} & =\mathbf{u}^{T} \cdot\left(\mathbf{i}-\mathbf{v} \frac{p_{u}}{v^{2}}\right)=\mathbf{u}^{T} \cdot \mathbf{i}-\left(\mathbf{u}^{T} \cdot \mathbf{v}\right) \frac{p_{u}}{v^{2}} \\
& =p_{u}-v^{2} \frac{p_{u}}{v^{2}} \equiv 0
\end{aligned}
$$

(i.e., $\mathbf{i}_{p 0}$ is parallel to $\mathbf{v}$, and $\mathbf{i}_{q 0}$ is orthogonal to $\mathbf{u}$ ).

Property 3: $\mathbf{i}_{0}$ is a vector component of $\mathbf{i}_{q 0}$, but not of $\mathbf{i}_{p 0}$. If $\mathbf{i}_{q 0}=0$, then the norm $i=\sqrt{ }\left(i_{A}^{2}+i_{B}^{2}+i_{C}^{2}\right)$ is, in general, not minimal for transmitting the instantaneous active power $p_{u}$, but the zero-sequence component, $\mathbf{i}_{0}$, is controlled to zero.

From the definitions and properties previously discussed, we can reach the following conclusions:

a) The current vector $\mathbf{i}_{p 0}$, component of the current vector $\mathbf{i}$, is indispensable for the instantaneous active power $\left(p_{u}\right)$ transmission, whereas $\mathbf{i}_{q 0}$ does not contribute to it because

$$
p_{u}=\mathbf{u} \cdot \mathbf{i}=\mathbf{u} \cdot \mathbf{i}_{p 0} \quad \text { and } \quad \mathbf{u} \cdot \mathbf{i}_{q 0} \equiv 0 .
$$

b) Current vector $\mathbf{i}_{p 0}$ transfers to the load instantaneous power $p_{u}$ at voltage $\mathbf{v}$, having the minimum norm when $v_{0}=0$ or $i_{0}=0$ (i.e., when $p_{0}=0$ ).

c) Using active filters without energy storage as compensators, the instantaneous pseudo-reactive current and the zero-

\begin{tabular}{|c|c|c|c|c|}
\hline & Case 1 & Case 2 & Case 3 \\
\hline \multirow{3}{*}{$\begin{array}{l}\text { Source } \\
\text { (A) }\end{array}$} & $I_{\text {SA }}$ & 43.3 & 43.7 & 42.4 \\
\hline & $\mathrm{I}_{\mathrm{SB}}$ & 39.1 & 39.1 & 38.2 \\
\hline & $\mathrm{I}_{\mathrm{SC}}$ & 39.1 & 32.0 & 35.0 \\
\hline \multirow{3}{*}{$\begin{array}{l}\text { Load } \\
\text { (A) }\end{array}$} & $\mathrm{I}_{\mathrm{LA}}$ & 46.4 & 46.4 & 46.4 \\
\hline & $\mathrm{I}_{\mathrm{LB}}$ & 37.1 & 37.1 & 37.1 \\
\hline & $\mathrm{I}_{\mathrm{LC}}$ & 37.1 & 29.8 & 29.8 \\
\hline \multirow{3}{*}{$\begin{array}{l}\mathrm{AF} \\
\text { (A) }\end{array}$} & $I_{C A}$ & 4.4 & 4.3 & 6.2 \\
\hline & $\overline{I_{C B}}$ & 4.2 & 4.2 & 4.9 \\
\hline & $I_{\mathrm{CC}}$ & 4.2 & 4.0 & 6.6 \\
\hline \multirow{3}{*}{$\begin{array}{l}\text { Neutral } \\
\text { (A) }\end{array}$} & $\mathrm{I}_{\mathrm{Sn}}$ & 0.0 & 7.8 & 0.0 \\
\hline & $\mathrm{I}_{\mathrm{Ln}}$ & 9.3 & 14.4 & 14.4 \\
\hline & $I_{\mathrm{Cn}}$ & 9.3 & 8.7 & 14.4 \\
\hline \multicolumn{2}{|c|}{$\mathrm{VA}_{\mathrm{AF}} / \mathrm{VA} \mathrm{A}_{\text {Source }}$} & 0.1 & 0.1 & 0.15 \\
\hline
\end{tabular}
sequence component can be controlled to zero, and the instantaneous active power cannot be changed. The line losses are lowered, ensuring the same instantaneous power consumption of the actual load. Thus, the second approach provides flexibility in compensating for the neutral current in a three-phase four-wire system including the zero-sequence voltage.
TABLE I

SIMULATION RESULTS

d) For three-phase four-wire systems with $p_{0}=0$, and threephase three-wire systems, the first and second approaches are in coincidence (i.e., $\mathbf{i}_{p} \equiv \mathbf{i}_{p 0}$ and $\mathbf{i}_{q} \equiv \mathbf{i}_{q 0}$ ).

2) Control Strategy: According to the second approach, the instantaneous pseudo-reactive current was compensated using the compensation current

$$
\mathbf{i}_{C}=\mathbf{i}_{q 0}
$$

the instantaneous active power generated by the compensator is zero (i.e., according to (18) and property 2),

$$
p_{C}=\mathbf{u}^{T} \cdot \mathbf{i}_{C}=0
$$

and the supply-source current after instantaneous compensation verifies

$$
\mathbf{i}_{S}=\mathbf{i}_{L}-\mathbf{i}_{C}=\mathbf{i}_{p 0}=\frac{p_{u}}{v^{2}} \mathbf{v} .
$$

So (20) shows that the supply current after compensation is free of the zero-sequence current component. The neutral current can be eliminated after instantaneous compensation, maintaining a coincidence with the objectives and criteria of the instantaneous power theories presented in [8]-[10].

\section{Simulation Results of Practical CASES}

Simulation was performed by MathCAD2000 Professional using the simulation model of Fig. 3, with three unsymmetrical load resistances $\left(R_{L A}=3.87 \Omega, R_{L B}=R_{L C}=4.84 \Omega\right)$, which was selected from reference [9] for comparison purposes. The three-phase four-wire system under sinusoidal balanced $\left(U_{A}=U_{B}=U_{C}=127 \mathrm{~V}\right)$ or imbalanced $\left(U_{A}=U_{B}=127\right.$ $\mathrm{V}, U_{C}=102 \mathrm{~V}$ ) voltage sources was considered. In our case, the simulation conditions were chosen to compare the two criteria of the previous paragraph. Case one is in balanced source voltages with instantaneous compensation following the mentioned criteria (the results are the same for the two criteria), while cases two and three are in imbalanced source voltages with instantaneous compensation following criteria one and two, respectively.

Table I displays the simulation results for the cases considered. Fig. 4 shows the simulation waveforms for case one, and Fig. 5 shows the simulation waveforms for cases two and three. 

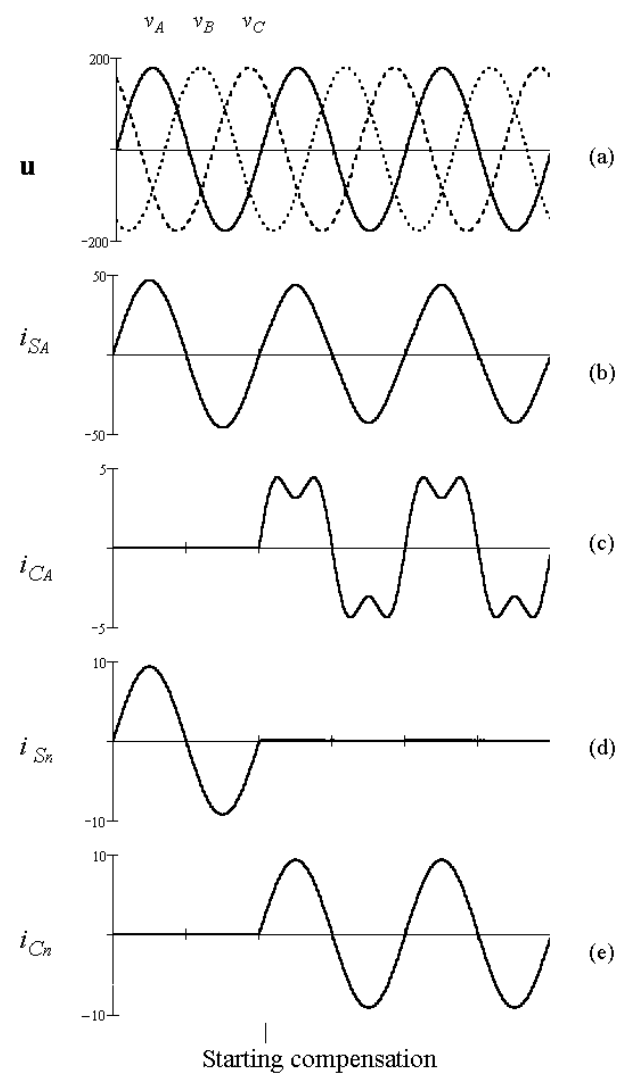

Fig. 4. Simulation results in case one; (a) balanced source voltages, (b) source currents of phase A, (c) compensating current of phase A, (d) neutral current on the source side, (e) neutral current of the active filter.

Each value in Table $I$ is measured at the peak point. The load currents in case one are imbalanced due to unsymmetrical load resistance. The load currents in cases two and three are imbalanced due to the unsymmetrical load resistance and the imbalanced source voltages. The ratios of the power rating of the active filter to that of the source are calculated based on the peak values.

Simulation waveforms in Cases two and three are shown in Fig. 5, indicated by solid and dashed lines, respectively. The source-side neutral current of case three, shown in Fig. 5(d), is perfectly controlled at zero. Now, as shown in Fig. 5(e), these neutral currents are changed to flow by the neutral line of the filter.

Finally, simulation results using Pspice have been included for a nonlinear load in star connection with the neutral wire, as shown in Fig. 6, under imbalanced voltage conditions. The voltage source includes a third-order harmonic (10\% of the fundamental). Each load leg, containing two antiparallel thyristors and series resistors, can be switched to obtain different load conditions. Fig. 7 shows illustrative waveforms for comparing results when the control strategies based on the studied approaches are applied to the active power filter. After reaching compensation, the load conditions were switched to simulate the process of instantaneous compensation and neutral current elimination.Fig. 7(a) and (b) show, according to the first approach, instantaneous compensation of the source and neutral currents. Cancellation of the neutral current on the source side is not com-

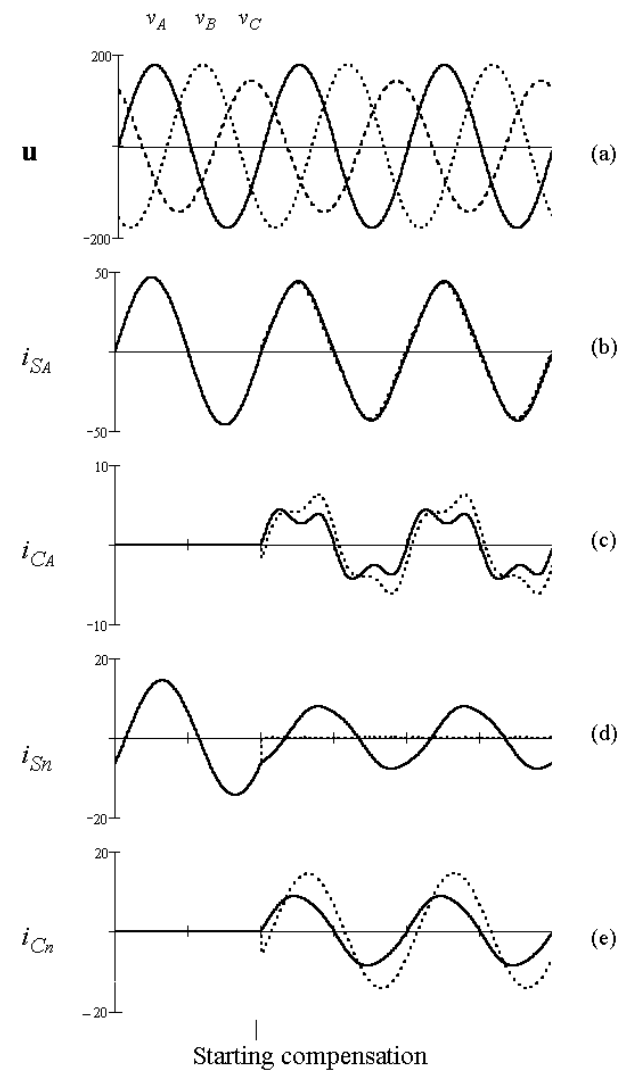

Fig. 5. Simulation results in case two (solid lines) and case three (dashed lines); (a) imbalanced source voltages, (b) source currents of phase A, (c) compensating currents of phase A, (d) neutral currents on the source side, (e) neutral currents of the active filter.

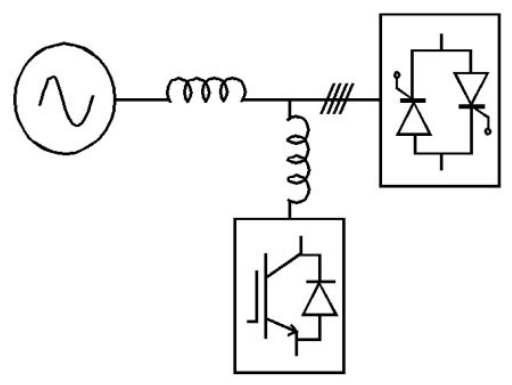

Fig. 6. Three-phase four-wire circuit with shunt active-power filter as reactive-power compensator.

plete. Fig. 7(c) and (d) show compensation of the source and neutral currents in the case of the second approach. When compensation starts, the neutral current in the source side is eliminated independently of the load value.

\section{CONCLUSIONS}

Vector decompositions [5] and [8] of the line current vector, according to the well-established instantaneous power concepts for three-phase systems, are reviewed in this paper. The present discussion is focused on three-phase four-wire load compensation when the zero-sequence voltage component exists. Two compensation criteria are discussed: 


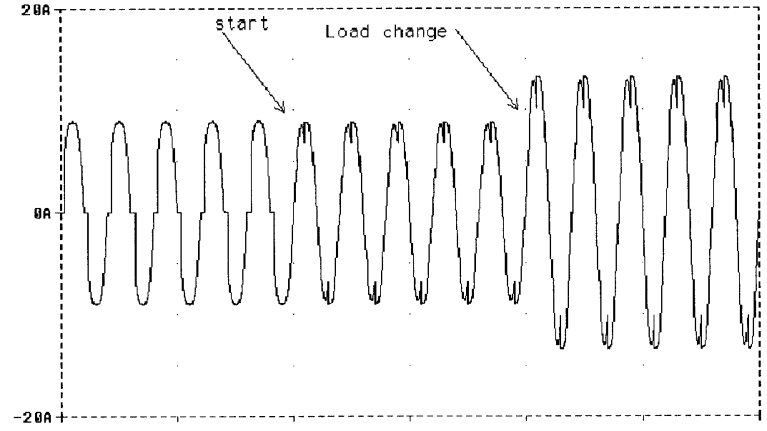

Time

(a)

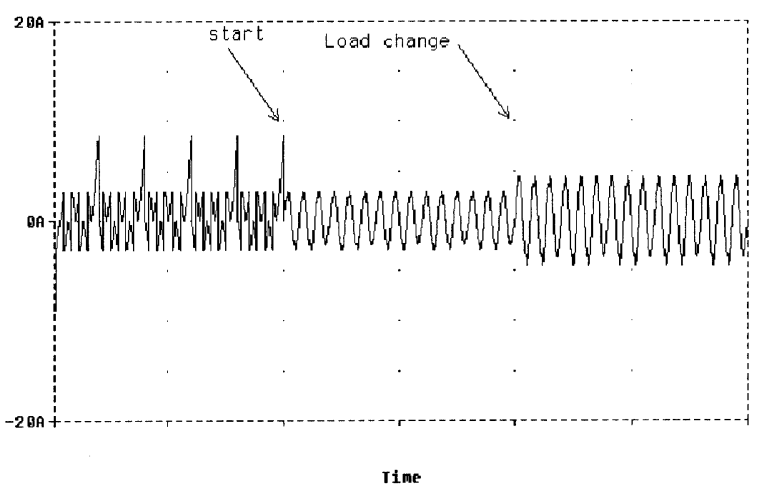

(b)

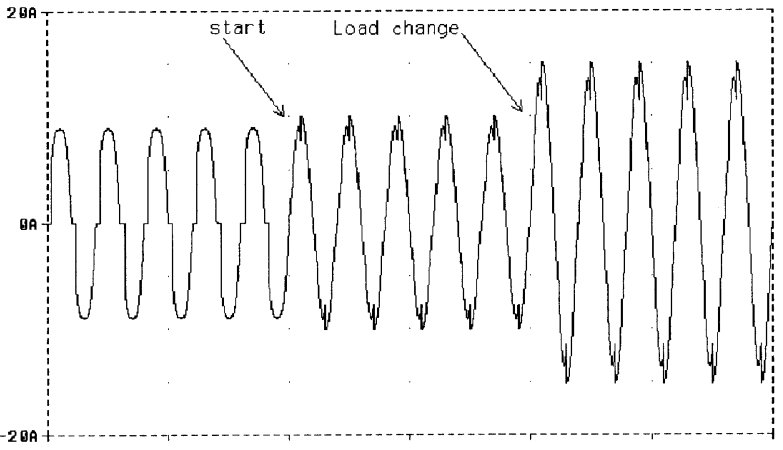

Time

(c)

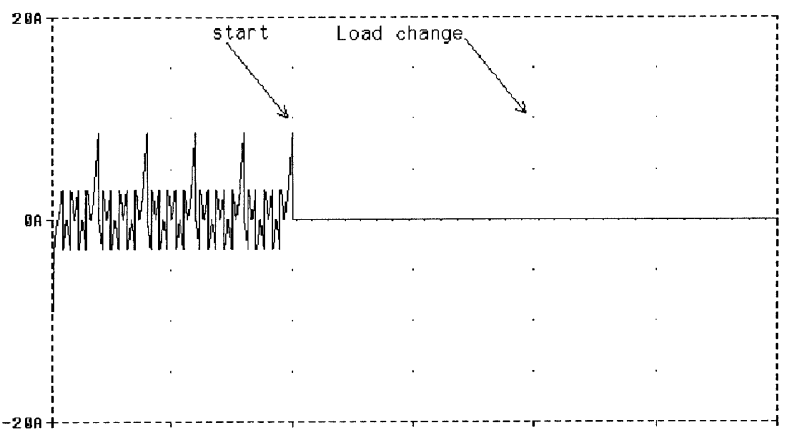

Time

(d)

Fig. 7. Waveforms before and after instantaneous compensation. (a) source current of phase A, and (b) neutral current on the source side, corresponding to the first approach. (c) Source current of phase A, and (d) neutral current on the source side, corresponding to the second approach.

1) for eliminating the instantaneous nonactive source-current component $\left(\mathbf{i}_{q}\right)$, which implies that zero-sequence current may still exist;

2) for eliminating part of the instantaneous nonactive source current component $\left(\mathbf{i}_{q 0}\right)$ and the zero-sequence current $\left(\mathbf{i}_{0}\right)$.

Thus, a compensating pseudocurrent is introduced, considering the points of view expressed in [5], [8], and [9]. Theory and computer simulation of different practical cases lead to the following conclusions for three-phase four-wire systems with existing zero-sequence components of voltage and current: a compensator without energy-storage components-an active filter-can fully compensate the neutral current when a proposed control strategy, based on the instantaneous pseudocurrents theory, is applied. However, the active filter cannot compensate for the neutral current fully, when a control strategy based on the well-established instantaneous power concepts [1]-[6] is applied.

Finally, the studied theories are perfectly identical if the zerosequence voltage is not included in three-phase systems.

\section{REFERENCES}

[1] H. Akagi, Y. Kanazawa, and A. Nabae, "Instantaneous reactive power compensators comprising switching devices without energy storage components," IEEE Trans. Ind. Applicat., vol. IA-20, pp. 625-630, May/June 1984
[2] L. Rossetto and P. Tenti, "Using Ac-fed PWM converters as instantaneous reactive power compensators," IEEE Trans. Power Electron., vol. 7, pp. 224-230, Jan. 1992.

[3] J. L. Willems, "A new interpretation of the Akagi-Nabae power components for nonsinusoidal three-phase situations," IEEE Trans. Instrum. Meas., vol. 41, pp. 523-527, Aug. 1992.

[4] A. Nabae and T. Tanaka, "A new definition of instantaneous active-reactive current and power based on instantaneous space vectors on polar coordinates in three-phase circuits," IEEE Trans. Power Delivery, vol. 11, pp. 1238-1243, July 1996.

[5] F. Z. Peng and J.-S. Lai, "Harmonic and reactive power compensation based on the generalized instantaneous reactive power theory for threephase four-wire systems," IEEE Trans. Power Electron., vol. 13, pp. 1174-1181, Nov. 1998.

[6] J. L. Willems, "Mathematical foundations of the instantaneous power concepts: A geometrical approach," Electromagnetic Transients Programs, vol. 6, pp. 299-303, Sept./Oct. 1996.

[7] P. Salmerón and J. C. Montaño, "Instantaneous power components in polyphase systems under nonsinusoidal conditions," Proc. Inst. Elect. Eng. Sci. Meas. Technol., vol. 143, pp. 151-155, Mar. 1996.

[8] J. C. Montaño and P. Salmerón, "Instantaneous and full compensation in three-phase systems," IEEE Trans. Power Delivery, vol. 13, pp. 1342-1347, Oct. 1998

[9] H. Kim and H. Akagi, "The instantaneous power theory based on mapping matrices in three-phase four-wire systems," in Proc. IEEE/IEE Japan Power Conv. Conf., Nagaoka, 1997, pp. 361-366.

[10] H. Akagi, Ogasawara, and H. Kim, "The theory of instantaneous in three-phase four-wire systems: A comprehensive approach," in IEEE IAS Annu. Meeting, 1999, pp. 431-439. 


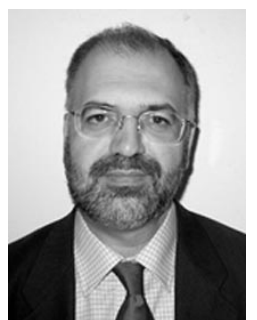

Juan Carlos Montaño Asquerino (M'80-SM'00) was born in Sanlúcar (Cádiz), Spain. He received the Ph.D. degree in physics from the University of Seville, Spain, in 1972.

Since 1978, he has been responsible for various projects in connection with research in power theory of nonsinusoidal systems, reactive power control, and power quality at the IRNAS (CSIC), Sevilla, Spain. From 1973 to 1978, he was a Researcher at the Instituto de Automática Industrial (CSIC-Spanish Research Council), Madrid, Spain, working on analog signal processing, electrical measurements, and control of industrial processes.

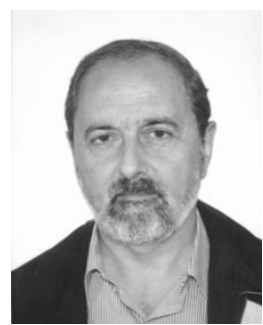

Patricio Salmerón Revuelta was born in Huelva, Spain. He received the Ph.D. degree in physics from the University of Seville, Spain, in 1993.

Currently, he is Professor of electric circuits and power electronics with the Escuela Politécnica Superior, Department of Electrical Engineering, University of Huelva, Spain, where he is Head of the department. From 1983 to 1993 , he was with the Department of Electrical Engineering, University of Seville, Spain. He has participated in various projects in connection with research in power theory of nonsinusoidal systems and power control in electrical systems. Presently, his research includes electrical power theory, active power filters, and artificial neural networks (ANNs). 